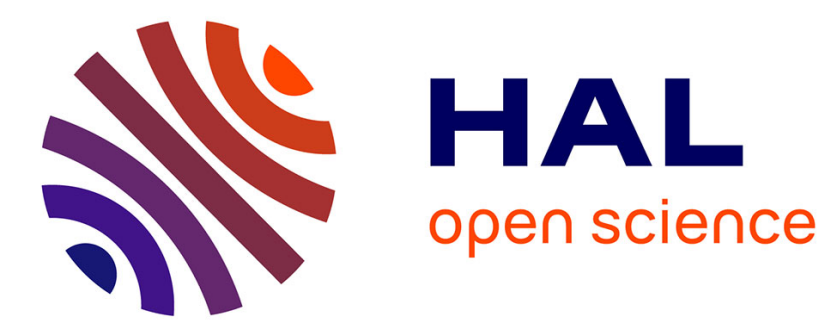

\title{
UAV-assisted disaster management: Applications and open issues
}

Milan Erdelj, Enrico Natalizio

\section{To cite this version:}

Milan Erdelj, Enrico Natalizio. UAV-assisted disaster management: Applications and open issues. International Conference on Computing, Networking and Communications (ICNC 2016), Feb 2016, Kauai, United States. pp.1-5, 10.1109/ICCNC.2016.7440563 . hal-01305371

\section{HAL Id: hal-01305371 \\ https://hal.science/hal-01305371}

Submitted on 21 Apr 2016

HAL is a multi-disciplinary open access archive for the deposit and dissemination of scientific research documents, whether they are published or not. The documents may come from teaching and research institutions in France or abroad, or from public or private research centers.
L'archive ouverte pluridisciplinaire HAL, est destinée au dépôt et à la diffusion de documents scientifiques de niveau recherche, publiés ou non, émanant des établissements d'enseignement et de recherche français ou étrangers, des laboratoires publics ou privés. 


\title{
UAV-Assisted Disaster Management: Applications and Open Issues
}

\author{
Milan Erdelj and Enrico Natalizio \\ Laboratory HEUDIASYC \\ University of Technology of Compiègne, France \\ \{milan.erdelj, enrico.natalizio\}@ @ds.utc.fr
}

\begin{abstract}
The fast-paced development of Unmanned Aerial Vehicles (UAVs) and their use in different domains, opens a new paradigm on their use in natural disaster management. In UAV-assisted disaster management applications, UAVs not only survey the affected area but also assist in establishing the communication network between the disaster survivors, rescue teams and nearest available cellular infrastructure. This paper identifies main disaster management applications of UAV networks and discusses open research issues related to UAVassisted disaster management.
\end{abstract}

\section{INTRODUCTION}

Large-scale natural disasters test the most fundamental human instinct of survival by inflicting massive, and often unpredictable loss to life and property. Various types of natural disasters, such as geophysical (earthquake, tsunami, volcano, landslide, avalanche), hydrological (flash-floods, debris flow, floods), climatological (extreme temperature, drought, wildfire) and meteorological (tropical storm, hurricane, sandstorm, heavy rainfall), among others, have caused losses of many lives in addition to increase in material losses in the order of $100 \%-150 \%$ over the period of last 30 years [1]. Acknowledging the need for bolstering disaster resilience, this paper describes a vision of leveraging the latest advances in wireless sensor network (WSN) technology and unmanned aerial vehicles (UAVs) to enhance the ability of networkassisted disaster prediction, assessment and response.

The occurrence of natural disasters is an important problem in all the areas of the world, both developed and developing. The problem arises from the physical extent of the disaster, which makes it out of scope of human possibilities to react to it. Currently, efforts are being done in order to recognize and forecast the possibility that a disaster will happen, to react in an efficient manner to the disaster in course of happening, and to quickly and efficiently assess the damage, fix and restore normal state. In this paper, we present different applications of UAV-assisted disaster management systems, and discuss about open issues and research challenges facing them.

The remainder of the paper is organized as follows: disaster management stages are presented in Section II, UAV-assisted disaster management applications in Section III and open issues and challenges in Section IV. Conclusions are drawn in Section V.

\section{DisASTER MANAGEMENT STAGES}

Natural disasters happen daily worldwide and represent an important factor that affects human life and development. In order to respond to different types of natural disasters and develop a feasible disaster management techniques and methods, it is important to understand the nature of a disaster, its phases and constituents.

When a disaster occurs, the most important issue that needs to be solved is to preserve human lives. In this context, the first 72 hours after the disaster hit are the most critical, which means that Search and Rescue (SAR) operations must be conducted quickly and efficiently. The International Search and Rescue Advisory Group (INSARAG) provides an international SAR protocol and methodology and publishes a set of guidelines ${ }^{1}$ which states that the SAR process must be conducted by teams. Activity assignment and local decisions are brought by a team leader, while all the team activities are coordinated by an incident commander. A common SAR mission is conducted in four major steps: 1) the commander establishes the search area (a smaller search area minimize the problems of communication among the rescuers), 2) establishing of a command post in the search area, 3) first responders are divided into scouts and rescuers and 4) scout teams report their findings to the command post and rescuers gather the information from the command post in order to know where to act.

In this paper, we envision a three-stage operational lifecycle where UAVs participate in natural disaster management:

- pre-disaster preparedness - concerning surveying-related events that precede the disaster, static WSN-based threshold sensing and setting up Early Warning Systems (EWS),

- disaster assessment - providing situational awareness during the disaster in real time and completing damage studies for logistical planning,

- disaster response and recovery - including SAR missions, forming the communications back-bone, insurancerelated field surveys.

Each stage imposes a set of task demands on the UAVs, lasts different lengths of time, and has varying priority levels.

\footnotetext{
${ }^{1}$ http://www.insarag.org/en/methodology/guidelines.html
} 


\section{UAV-ASSISTED DISASTER MANAGEMENT} APPLICATIONS

In this work, the classification of the WSN and UAVassisted applications in disaster management is based on the goal of the particular set of applications. For instance, the applications of structural monitoring, disaster forecast, environmental monitoring and early warning system design are grouped together since their common goal is to predict and forecast the occurrence of a disaster. WSN and UAVassisted applications in disaster management that are taken into account in this work are the following: (a) monitoring, forecast and early warning systems, (b) disaster information fusion, (c) situational awareness and logistics, (d) damage assessment, (e) standalone communication system, (f) search and rescue missions. The review of related works on using WSN and UAVs in disaster management is presented in Table I.

\section{A. Monitoring, forecast, early warning systems}

This section covers the applications of WSN and UAVs where the goal is to predict the disaster by structural and environmental monitoring, information analysis for forecasting and early warning systems. The goal of predicting and forecasting the natural disaster are executed during the prevention and preparedness phases of the disaster management cycle.

An early warning system for natural disasters, which relies on existing and available WSN technologies and that focuses on issues of providing reliable data transmission, important amounts of data from heterogeneous sensors and minimizing energy consumption is described in [5]. Methodology, techniques and integrated services adopted for the design and the realization of a web-based platform for automatic and continuous monitoring of the Rotolon landslide (Eastern Italian Alps) is tackled in [11]. The solution that employs UAVs to reduce problems that arise from faults in a sensor network during natural disaster is outlined in [30].

\section{B. Disaster information fusion}

Although information fusion is necessary and helpful in all the disaster management stages, its most important impact is seen in the assessment stage. The goal of the information fusion and knowledge sharing is to combine different sources of information available and/or to make a bridge between different information technologies that can be of use in other applications for disaster management. For instance, a first responder system that implements information fusion and that is based on the use of mobile autonomous agents that are deployed in the emergency area is proposed in [16].

An experimental system where multiple heterogeneous vehicles come together and are controlled and coordinated via cyberspace to accomplish a complex logistical operation in automated humanitarian missions is presented in [20]. In [3] and [14], authors propose an efficient architecture of a smart public safety platform that integrates heterogeneous components such as smart data gathering and analysis system, communication system, WSN and social networks in [3] and UAVs and Unmanned Ground Vehicles (UGVs) in [14].

\section{Situational awareness and logistics}

The goal of this set of applications in disaster management is to gather the information during the disaster phase, especially regarding the movement of the people endangered by the disaster, as well as the rescue teams deployed on the disaster area. The platform that focuses on both static and mobile sensors and addresses the challenges of sensor data aggregation, routing, responders activity monitoring and different mobility issues is presented in [8]. Experiences from La Conchita (California) mudslide response in January 2005 are described in [21], where authors provide a study about information regarding mudslide responses, what tasks robots are needed for, how the rescue robots performed and how responders viewed the robots. The solution for crisis management proposed in [27] is focused on tracking and data aggregation methods in order to gather the information about the fire status and its evolution, besides the tasks of safe-zone discovery and aggregation of rescue personnel biomedical signs. In [13], authors present DistressNet, an ad hoc wireless architecture that supports disaster response with distributed collaborative sensing, topology-aware routing using a multichannel protocol, and accurate resource localization.

\section{Damage assessment}

When a disaster happen, it is important to assess the scale of the damage by using different methods such as structural health monitoring and UAV video inspection. In this context, Kruijff et al. describe the deployment and experience in working with the Italian National Fire Corps involved in the post-disaster assessment on July, 2012, after two major earthquakes that occurred in Emilia-Romagna region in Northern Italy [15]. The conclusion brought after the mission is that UAV and UGV operator suffered from cognitive overload, which justifies the research in the field of automatic damage assessment systems. Key challenges to implement faulttolerant and efficient deployments of collaborative autonomous aircraft to increase operational reliability and performance when performing aerial sensing and assessment are described in [26]. In [9], authors discuss the use of a low-cost UAV-based remote sensing system for disaster assessment, environmental management and infrastructure development monitoring. Similarly, in [31], authors develop a small UAV system that is capable of acquiring aerial disaster assessment information.

\section{E. Standalone communication systems}

The application that receives the most attention during the disaster management is related to standalone communication systems, where the goal is to re-establish the damaged or destroyed communication infrastructure during the disaster. Its application is most relevant during the disaster response phase, while the re-established standalone communication system can be of use during the recovery phase as well. In order to facilitate the intercommunication between disaster victims and rescue teams, an integrated emergency communication system that relies on WSN is proposed in [2]. Similar concept is proposed in [4], [6], [12], [17], [19], [22] and [29], where 
TABLE I

UAV-ASSISTED DISASTER MANAGEMENT APPLICATIONS.

\begin{tabular}{|c|c|c|c|c|c|c|c|c|c|c|c|c|}
\hline \multicolumn{2}{|l|}{ Related works } & \multicolumn{3}{|c|}{ Disaster stages } & \multicolumn{2}{|c|}{ Technology } & \multicolumn{6}{|c|}{ UAV-assisted applications } \\
\hline Authors & Year & $\begin{array}{l}\text { Pre-disaster } \\
\text { preparedness }\end{array}$ & $\begin{array}{c}\text { Disaster } \\
\text { assessment }\end{array}$ & $\begin{array}{c}\text { Post-disaster } \\
\text { response \& recovery }\end{array}$ & WSN & UAV & $\begin{array}{l}\text { Monitoring, } \\
\text { forecast, EWS }\end{array}$ & $\begin{array}{l}\text { Information } \\
\text { fusion }\end{array}$ & $\begin{array}{l}\text { Situational } \\
\text { awareness }\end{array}$ & $\begin{array}{c}\text { Damage } \\
\text { assessment }\end{array}$ & $\begin{array}{c}\text { Standalone } \\
\text { comm. system }\end{array}$ & $\begin{array}{c}\text { SAR } \\
\text { missions }\end{array}$ \\
\hline Frigerio et al [11] & 2014 & $\bullet$ & & & $\bullet$ & & $\bullet$ & & & & & \\
\hline Ueyama et al [30] & 2014 & $\bullet$ & & $\bullet$ & $\bullet$ & $\bullet$ & $\bullet$ & & & & $\bullet$ & \\
\hline Erman et al [8] & 2008 & $\bullet$ & $\bullet$ & & $\bullet$ & $\bullet$ & $\bullet$ & & $\bullet$ & & & \\
\hline Chen et al [5] & 2013 & $\bullet$ & $\bullet$ & & $\bullet$ & & $\bullet$ & $\bullet$ & & & & \\
\hline Bartoli et al [3] & 2015 & $\bullet$ & $\bullet$ & & $\bullet$ & & & $\bullet$ & & & & \\
\hline Kumar et al [16] & 2004 & & $\bullet$ & & $\bullet$ & $\bullet$ & & $\bullet$ & & $\bullet$ & & \\
\hline Mosterman et al [20] & 2014 & & $\bullet$ & & $\bullet$ & $\bullet$ & & $\bullet$ & & & & \\
\hline Sardouk et al [27] & 2010 & & $\bullet$ & $\bullet$ & $\bullet$ & & & $\bullet$ & & & & $\bullet$ \\
\hline Grocholsky et al [14] & 2006 & & $\bullet$ & & & $\bullet$ & & & $\bullet$ & & & \\
\hline George et al [13] & 2010 & & $\bullet$ & & $\bullet$ & & & & $\bullet$ & & & \\
\hline Pogkas et al [25] & 2007 & & $\bullet$ & $\bullet$ & $\bullet$ & & & & $\bullet$ & & & $\bullet$ \\
\hline Murphy et al [21] & 2008 & & $\bullet$ & $\bullet$ & & $\bullet$ & & & $\bullet$ & & & $\bullet$ \\
\hline Wada et al [31] & 2013 & & $\bullet$ & & & $\bullet$ & & & & $\bullet$ & & \\
\hline Ezequiel et al [9] & 2014 & & $\bullet$ & & & $\bullet$ & & & & $\bullet$ & & \\
\hline Kruijff et al [15] & 2012 & & $\bullet$ & $\bullet$ & & $\bullet$ & & & & $\bullet$ & & $\bullet$ \\
\hline Fujiwara and Watanabe [12] & 2005 & & & $\bullet$ & $\bullet$ & & & & & & $\bullet$ & \\
\hline Bai et al [2] & 2010 & & & $\bullet$ & $\bullet$ & & & & & & $\bullet$ & \\
\hline Fragkiadakis et al [10] & 2011 & & & $\bullet$ & $\bullet$ & & & & & & $\bullet$ & \\
\hline Nelson et al [22] & 2011 & & & $\bullet$ & & $\bullet$ & & & & & $\bullet$ & \\
\hline Tuna et al [29] & 2012 & & & $\bullet$ & $\bullet$ & $\bullet$ & & & & & $\bullet$ & \\
\hline Morgenthaler et al [19] & 2012 & & & $\bullet$ & & $\bullet$ & & & & & $\bullet$ & \\
\hline Dalmasso et al [6] & 2012 & & & $\bullet$ & & $\bullet$ & & & & & $\bullet$ & \\
\hline Marinho et al [17] & 2013 & & & $\bullet$ & & $\bullet$ & & & & & $\bullet$ & \\
\hline Minh et al [18] & 2014 & & & $\bullet$ & $\bullet$ & & & & & & $\bullet$ & \\
\hline Carli et al [4] & 2014 & & & $\bullet$ & $\bullet$ & & & & & & $\bullet$ & \\
\hline Di Felice et al [7] & 2014 & & & $\bullet$ & & $\bullet$ & & & & & $\bullet$ & \\
\hline Robinson and Lauf [26] & 2013 & & & $\bullet$ & & $\bullet$ & & & & & $\bullet$ & $\bullet$ \\
\hline Nourbakhsh et al [23] & 2005 & & & $\bullet$ & $\bullet$ & & & & & & & $\bullet$ \\
\hline Tuna et al [28] & 2014 & & & $\bullet$ & $\bullet$ & $\bullet$ & & & & & & $\bullet$ \\
\hline
\end{tabular}

the network of UAVs acts as a backbone of a communication network damaged during a disaster.

Another flexible network architecture that provides a common networking platform for heterogeneous multi-operator networks, for interoperation in case of emergencies is proposed in [10]. Authors measured the performance of a video streaming application in a real wireless metropolitan multiradio mesh network, showing that the mesh network can meet the requirements for high quality video transmissions [24]. A distributed mobility algorithm for re-establishing connectivity among the disconnected end-user in post-disaster scenarios is proposed in [7]. The architecture proposed in [18] extends Internet connectivity from surviving access points to disaster victims using their own mobile devices and it is set up on demand using wireless virtualization to create virtual access points on mobile devices.

\section{F. Search and rescue missions}

The goal of this set of WSN and UAV-assisted applications is to search for and to rescue the misfortunate people that happen to be lost, trapped by debris or injured during the disaster or immobile by any other means. An architecture for urban SAR and a methodology for mixing real-world and simulation-based testing, where a sensor suite and sensor fusion algorithm for victim detection permits aggregation of sensor readings from various sensors on multiple robots is provided in [23]. A similar ad-hoc sensor network for disaster relief applications that provides rescue teams with a quickly deployable and reliable tool to collect information about the presence of people in a collapsed building is presented in [25]. Approach to WSN deployment with the use of mobile robots for human existence detection in case of disasters is shown in [28].

\section{G. Other applications}

Health and wellbeing of people are among the most critical issues that an efficient disaster management system should take care of. Therefore, different medical applications are widespread prior, during and after the disaster happened. It includes the problems of mitigating the disease risks triggered by the disaster, as well as the first aid and supply delivery. Finally, the use of WSN and UAVs in infrastructural reconstruction is relied on during the stage of recovery after the disaster, where the goal is to improve the construction process that will allow the "smart" construction of infrastructure taking into account the knowledge gained in prior disaster occurrences. 


\section{OPEN ISSUES}

Involving UAVs in disaster management have several networking-related research challenges, as described below:

\section{A. UAV Localization}

Given their limited flying time and the need for time-critical action, allowing UAVs to autonomously scout the disaster area and select their locations with self-learning techniques using repeated trials may be sub-optimal. We advocate the use of partial external inputs to guide the UAVs in establishing the last-hop connectivity to users as well as establishing a relay network. Last recorded information from cellular network location database as well as pings from mobile devices can be leveraged to estimate the density of affected people and their geographical distribution, which requires new signaling protocols between operators. Approaches like ant-foraging algorithms that reinforce paths based on availability/number of mobile pings can drive the UAVs towards the locations with high density of survivors.

\section{B. Creating and maintaining the relay network}

The relaying network formed by the UAVs is completely aerial and must have a high level of resilience towards link outages owing to motion-related changes or energy-level changes among the UAVs. A two stage process is needed an initial round of centralized determination of optimal relay points (which we call anchors) that connect the disaster region to the nearest radio access network is followed by a round of de-centralized correction during deployment. The problem of allocating backup UAVs for each anchor point is analogous to reserving backup channels in cellular environments, with several major differences: the ability of the backup UAVs to serve as relay-anchors may change over time depending on energy level variations; the handoff process itself consumes resources in terms of movement towards the anchor location; the functional loss that results in altering the role of the UAV from surveying to relaying, among others.

Interesting problems include: (i) performing optimal handoffs between the roles of surveying, communication with users, and data relaying, (ii) choosing the charging duration, i.e., making tradeoff decisions on whether charging instants should be proactive, even if their battery is not completely depleted, (iii) optimizing the number of hops by building accurate 3D channel models for various weather conditions and land topologies, (iv) designing distributed control algorithms at different layers of the UAV-assisted disaster management system, etc.

\section{Data fusion and handover issues}

The video/images collected by the UAVs present an overview of the situation. However, affected humans may also use various social media or forward text messages and images via the UAV relay network. These offer fine-grained on-theground information that can be fused at the control center with the high definition UAV feeds. In-network data fusion within a mobile UAV network with energy constraints has not yet been investigated thoroughly. Additionally, the need for data fusion can impact the UAV network in interesting ways: (i) a more complete view of the situation can direct UAVs towards specific regions, (ii) it can reduce the data forwarding requirements from the UAVs, thereby saving more energy for flight. Existing source/channel coding from the domain of multimedia sensor networks are not sufficient as they consider a static network topology with varying channel conditions. Here, both topology and the channel changes with time.

The handover process among UAVs can begin early, during the approach time of the UAV towards the designated location, though this involves higher transmission power and increased impact of the 3-D propagation environment. Additionally, the simultaneous actions of movement in air and RF transmissions may introduce signal fluctuations caused by Doppler effect at the incoming UAV. On the other end, UAVs can align themselves next to each other in air, and then begin the handover. However, there is a tradeoff between the advantage of aerial stability during handover-related messaging with low transmission power, and the correspondingly lengthy duration for completing the entire handover process.

\section{UAV-assisted system sustainability}

The majority of UAVs have about 20-30 mins of flight time, and one way to extend the network lifetime is to alternate network responsibilities among them. Some parallels with techniques to manage networked nodes in the Bluetooth standard can be drawn here, such as the master node placing selected slave nodes in parked mode, once the number of active connections increases beyond 7 . Works on moving Bluetooth slaves between active and parked modes based on energy availability and anticipated traffic needs can provide some preliminary strategies, though these will need to be majorly extended for the UAV scenario. However, the parking action in the UAV context is not merely switching the role status to an inactive mode. Instead, it involves complex spatio-temporal coordination of identifying safe stationary locations for the UAV to land on-ground and perform other non-aerial tasks. For example, redundant on-ground UAVs can serve as groundrelay nodes connecting to the aerial mesh layer, or fixed base stations serving mobile handsets, without consuming energy for continuous flight (Figure 1).

The location of charging points as well as the energy transfer methods must be carefully optimized as this impacts the duration of active service. Magnetic-induction based charging plates installed on on the supporting ground vehicles is one feasible option, which allow the UAVs to land and re-charge without manual intervention. This also raises new possibilities of joint optimization and practical applications of existing research on target tracking, where the vehicles are mobile and can position themselves in optimal locations, such as around the periphery of the affected area, to minimize the UAV travel time from its designated operational area to the selected charging point. 


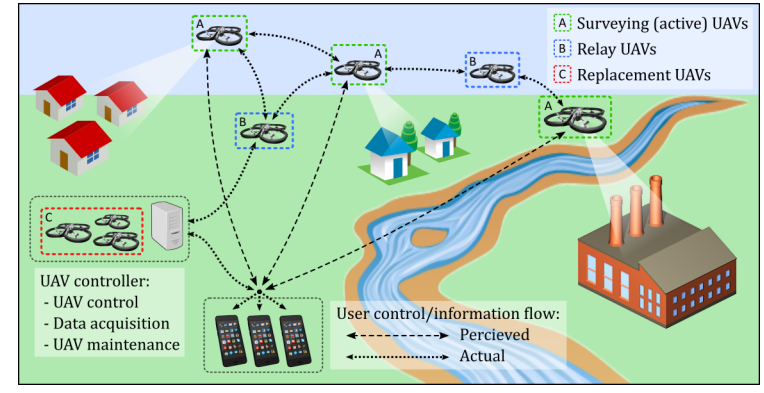

Fig. 1. Illustration of a UAV-assisted disaster management system.

\section{CONCLUSION}

This paper identified main disaster management applications of UAV networks and discusses open research issues related to the use of UAVs. Based on the surveyed related works, UAV networks in conjunction with WSN and cellular network are showed to be a promising future technology for the applications in disaster management.

\section{ACKNOWLEDGEMENTS}

This work has been carried out in the framework of the project "IMATISSE" (Inundation Monitoring and Alarm Technology In a System of SystEms), which is funded by the Region Picardie, France, through the European Regional Development Fund (ERDF).

\section{REFERENCES}

[1] Münchener Rückversicherungs-Gesellschaft. Loss Events Worldwide 1980- 2014. Geo Risks Research, NatCatSERVICE, 2015.

[2] Y. Bai, W. Du, Z. Ma, C. Shen, Y. Zhou, and B. Chen. Emergency communication system by heterogeneous wireless networking. In Wireless Communications, Networking and Information Security (WCNIS), 2010 IEEE International Conference on, June 2010.

[3] G. Bartoli, R. Fantacci, F. Gei, D. Marabissi, and L. Micciullo. A novel emergency management platform for smart public safety. International Journal of Communication Systems, 2015.

[4] M. Carli, S. Panzieri, and F. Pascucci. A joint routing and localization algorithm for emergency scenario. Ad Hoc Networks, 2014.

[5] D. Chen, Z. Liu, L. Wang, M. Dou, J. Chen, and H. Li. Natural disaster monitoring with wireless sensor networks: A case study of dataintensive applications upon low-cost scalable systems. Mobile Networks and Applications, 2013.

[6] I. Dalmasso, I. Galletti, R. Giuliano, and F. Mazzenga. WIMAX networks for emergency management based on UAVs. In Satellite Telecommunications (ESTEL), 2012 IEEE First AESS European Conference on, 2012.

[7] M. Di Felice, A. Trotta, L. Bedogni, K. R. Chowdhury, and L. Bononi. Self-organizing aerial mesh networks for emergency communication. In Personal, Indoor, and Mobile Radio Communication (PIMRC), 2014 IEEE 25th Annual International Symposium on, 2014.

[8] A. Erman, L. Hoesel, P. Havinga, and J. Wu. Enabling mobility in heterogeneous wireless sensor networks cooperating with UAVs for mission-critical management. Wireless Communications, IEEE, 2008.

[9] C. Ezequiel, M. Cua, N. Libatique, G. Tangonan, R. Alampay, R. Labuguen, C. Favila, J. Honrado, V. Canos, C. Devaney, A. Loreto, J. Bacusmo, and B. Palma. UAV aerial imaging applications for postdisaster assessment, environmental management and infrastructure development. In Unmanned Aircraft Systems (ICUAS), 2014 International Conference on, 2014.

[10] A. G. Fragkiadakis, I. G. Askoxylakis, E. Z. Tragos, and C. V. Verikoukis. Ubiquitous robust communications for emergency response using multi-operator heterogeneous networks. Eurasip Journal on Wireless Communications and Networking, 2011.
[11] S. Frigerio, L. Schenato, G. Bossi, M. Cavalli, M. Mantovani, G. Marcato, and A. Pasuto. A web-based platform for automatic and continuous landslide monitoring: The Rotolon (eastern Italian Alps) case study. Computers and Geosciences, 2014.

[12] T. Fujiwara and T. Watanabe. An ad hoc networking scheme in hybrid networks for emergency communications. Ad Hoc Networks, 2005.

[13] S. George, W. Zhou, H. Chenji, M. Won, Y. O. Lee, A. Pazarloglou, R. Stoleru, and P. Barooah. DistressNet: A wireless ad hoc and sensor network architecture for situation management in disaster response. Communications Magazine, IEEE, 2010.

[14] B. Grocholsky, J. Keller, V. Kumar, and G. Pappas. Cooperative air and ground surveillance. Robotics Automation Magazine, IEEE, 2006.

[15] G.-J. Kruijff, V. Tretyakov, T. Linder, F. Pirri, M. Gianni, P. Papadakis, M. Pizzoli, A. Sinha, E. Pianese, S. Corrao, F. Priori, S. Febrini, and S. Angeletti. Rescue robots at earthquake-hit Mirandola, Italy: A field report. In Safety, Security, and Rescue Robotics (SSRR), 2012 IEEE International Symposium on, 2012.

[16] V. Kumar, D. Rus, and S. Singh. Robot and sensor networks for first responders. Pervasive Computing, IEEE, 2004.

[17] M. Marinho, E. de Freitas, J. Lustosa da Costa, A. de Almeida, and J. de Sousa, R.T. Using cooperative mimo techniques and UAV relay networks to support connectivity in sparse wireless sensor networks. In Computing, Management and Telecommunications (ComManTel), 2013 International Conference on, 2013.

[18] Q. T. Minh, K. Nguyen, C. Borcea, and S. Yamada. On-the-fly establishment of multihop wireless access networks for disaster recovery. Communications Magazine, IEEE, 2014.

[19] S. Morgenthaler, T. Braun, Z. Zhao, T. Staub, and M. Anwander. UAVNet: A mobile wireless mesh network using unmanned aerial vehicles. In Globecom Workshops (GC Wkshps), 2012 IEEE, 2012.

[20] P. Mosterman, D. Sanabria, E. Bilgin, K. Zhang, and J. Zander. A heterogeneous fleet of vehicles for automated humanitarian missions. Computing in Science Engineering, 2014.

[21] R. R. Murphy and S. Stover. Rescue robots for mudslides: A descriptive study of the 2005 la Conchita mudslide response. Journal of Field Robotics, 2008.

[22] C. Nelson, B. Steckler, and J. Stamberger. The evolution of hastily formed networks for disaster response: Technologies, case studies, and future trends. In Global Humanitarian Technology Conference (GHTC), 2011 IEEE,2011.

[23] I. R. Nourbakhsh, K. Sycara, M. Koes, M. Yong, M. Lewis, and S. Burion. Human-robot teaming for search and rescue. IEEE Pervasive Computing, 2005.

[24] M. Pinson, S. Wolf, and R. Stafford. Video performance requirements for tactical video applications. In Technologies for Homeland Security, 2007 IEEE Conference on, 2007.

[25] N. Pogkas, G. Karastergios, C. Antonopoulos, S. Koubias, and G. Papadopoulos. Architecture design and implementation of an ad-hoc network for disaster relief operations. Industrial Informatics, IEEE Transactions on, 2007.

[26] W. Robinson and A. Lauf. Resilient and efficient MANET aerial communications for search and rescue applications. In Computing, Networking and Communications (ICNC), 2013 International Conference on, 2013.

[27] A. Sardouk, M. Mansouri, L. Merghem-Boulahia, D. Gaiti, and R. Rahim-Amoud. Multi-agent system based wireless sensor network for crisis management. In Global Telecommunications Conference (GLOBECOM 2010), 2010 IEEE, 2010.

[28] G. Tuna, V. C. Gungor, and K. Gulez. An autonomous wireless sensor network deployment system using mobile robots for human existence detection in case of disasters. Ad Hoc Networks, 2014.

[29] G. Tuna, T. Mumcu, and K. Gulez. Design strategies of unmanned aerial vehicle-aided communication for disaster recovery. In High Capacity Optical Networks and Enabling Technologies (HONET), 2012 9th International Conference on, 2012.

[30] J. Ueyama, H. Freitas, B. Faical, G. Filho, P. Fini, G. Pessin, P. Gomes, and L. Villas. Exploiting the use of unmanned aerial vehicles to provide resilience in wireless sensor networks. Communications Magazine, IEEE, 2014

[31] A. Wada, T. Yamashita, M. Maruyama, T. Arai, H. Adachi, and H. Tsuji. A surveillance system using small unmanned aerial vehicle (UAV) related technologies. NEC Technical Journal, 2013. 